\title{
Contact tracing in Lassa fever Outbreak Response, an effective strategy for control?
}

\author{
Joan Ejembi ${ }^{1,2}$, Uloaku Emma-Ukaegbu², Itopa Garba ${ }^{2}$, Anthony Omale ${ }^{2}$, Bala Dogo ${ }^{2}$, Lydia \\ Taiwo 2 \\ ${ }^{1}$ Medical Microbiology, Ahmadu Bello University, Zaria, Benue, Nigeria, ${ }^{2}$ Nigeria Field Epidemiology and Laboratory Training Programme, Abuja, \\ Federal Capital Territory, Nigeria \\ Objective
}

To identify risk factors among contacts of Lassa fever cases which can predispose to disease spread and institute control measures. Introduction

Contact tracing is an important strategy employed in surveillance to aid prompt detection of infectious disease and control outbreaks. It involves the identification of those who have come in contact with an infectious person and following them up for the duration of the incubation period of the disease to promptly detect symptoms and signs and institute treatment thus reducing chances of disease spread to other susceptible individuals. It is a primary means of disease prevention. The importance of cooperation from contacts being traced cannot be overemphasized as they are required to promptly report symptoms, avoid gatherings and travelling until they are cleared by the surveillance team. The follow-up should also link contacts who become symptomatic to designated care centers. In 2014, during the Ebola outbreak in Nigeria, the disease spread from Lagos to another State in the country by a contact who travelled out of Lagos to Rivers state.

To identify risk factors among contacts of Lassa fever cases which can predispose to disease spread and institute control measures, we interviewed primary contacts of Lassa fever cases during an outbreak response in Kogi State Nigeria, 2016

\section{Methods}

We Identified contacts of Lassa fever cases (confirmed/suspected/probable) among family, friends, community members, coworkers and health care workers, followed up for 21 days during a Lassa fever outbreak which started in February 2016 at Kogi State Nigeria. Contacts were interviewed using a structured interviewer administered questionnaire with sections on demography, risk factors for infection and spread of Lassa fever, symptoms developed during the follow-up period and adherence to prorocol. Control measures were instituted to address identified gaps. We defined a contact as anyone irrespective of age, occupation or sex who came in contact with any of the cases of Lassa fever classified as either confirmed/suspected/probable and used standard IDSR case definitions for Suspected, Confirmed and Probable cases of Lassa fever. Data was analysed with Epi Info version 7

\section{Results}

Overall 149 contacts were interviewed, $79(53.0 \%)$ were female, the mean age of respondents was $33.2+-10.1$ and many were health care workers 61(40.9\%). Of the respondents, $18(12.0 \%)$ live or work in areas infested with rodents, $21(14.1 \%)$ ate bush meat, $2(2.5 \%)$ of the females were pregnant and $20(13.4 \%)$ of respondents travelled out of station with $1(5 \%)$ contact crossing international borders. During the follow-up period, $14(9.4 \%)$ developed symptoms suggestive of Lassa fever. Of these $12(85.7 \%)$ sought treatment and the options were self-medication $3(25.0 \%)$ and presenting at a health facility $9(77.8 \%)$. The health facilities visited were mainly privately owned $7(77.9 \%)$ and only $1(11.1)$ was a tertiary health care facility. We instituted the following interventions; Health education of contacts and linkage of symptomatic contacts to the designated treatment center where treatment commenced and samples were collected and sent to reference lab for diagnosis. All samples 14(100\%) came back negative for Lassa fever. 


\section{Conclusions}

Contact tracing is an important strategy in controlling outbreaks of infectious diseases. However, risk factors identified among contacts can hinder the effectiveness of this strategy and facilitate spread of the disease. We recommend training on health education and Lassa fever transmission for contacts of cases and the need to adhere to protocol so that the ultimate aim of interrupting transmission can be achieved.

\section{Acknowledgement}

Nigerian Field Epidemiology and Laboratory Training Programme State Ministry Of Health Kogi State,Nigeria Nigeria Center for Disease Control

\section{References}

1. Nigeria Center for Disease Control. Viral Haemorrhagic Fevers, Preparedness and Response Plan. 2017 